\title{
Ciclo e-M: Avaliação Sistemática da Gestão Tecnopedagógica
}

\author{
Herik Zednik Rodrigues ${ }^{1}$, Liane Margarida Rockenbach Tarouco ${ }^{1}$, Luis Roque \\ Klering $^{2}$, Eder Paulus M. Guerra ${ }^{3}$ \\ ${ }^{1}$ Programa de Pós-graduação em Informática na Educação - Universidade Federal do \\ Rio Grande do Sul (UFRGS) \\ ${ }^{2}$ Escola de Administração - Universidade Federal do Rio Grande do Sul (UFRGS) \\ ${ }^{3}$ Universidade Estadual Vale do Acaraú (UVA) \\ herik.zednik@ufrgs.br, liane@penta.ufrgs.br, lrklering@via-rs.net, \\ ederpaulus yahoo.com.br
}

\begin{abstract}
The paper aims to present and describe the phases of the Cycle e-M. The methodology used is a theoretical and analytics approach based on the model CM 360 , proposed by Franco, Santos and Terra (2009). That cycle comprises the model e-Maturity: Technopedagogical management, which is characterized by a questionnaire type web-based self-assessment to monitor the level of technological maturity. The Cycle e-M proposes systematic investigation of seven dimensions (Management and Leadership, Curriculum Planning, Process of Teaching and Learning, Assessment, Training for use of ICT in Education, Resources and e-safety) and is divided into four distinct phases: Action, Assessment, Analysis and Planning.
\end{abstract}

Resumo. $O$ artigo objetiva apresentar e descrever as fases do Ciclo e-M. A metodologia utiliza-se de uma abordagem teórico-analítica fundamentada no modelo CM $360^{\circ}$, proposto por Franco, Santos e Terra (2009). O referido ciclo compõe o modelo e-Maturity: Gestão Tecnopedagógica, que se caracteriza por ser um questionário do tipo web-based de autoavaliação para acompanhamento do nível de maturidade tecnológica. O Ciclo e-M propõe averiguação sistemática de 7 dimensões (Gestão e Liderança; Planejamento Curricular; Processo de Ensino e Aprendizagem; Avaliação; Formação Profissional para uso das TIC na Educação; Recursos e e-segurança) e divide-se em 4 fases distintas: Ação, Avaliação, Análise e Planejamento.

\section{Introdução}

Articular a realidade de uma escola com o domínio didático e administrativo dos recursos tecnológicos e a prática profissional com as Tecnologias da Informação e da Comunicação (TIC) é o grande desafio do gestor escolar da atualidade. O crescente aumento do volume de tecnologias disponíveis no ambiente escolar resulta na necessidade de auxiliar de forma sistemática gestores e comunidade escolar sobre como usar avaliações para melhorar sua prática de gestão da tecnologia, de forma que esta se reflita positivamente no processo de ensino e aprendizagem.

Parte-se do pressuposto de que as "[...] avaliações são instrumentos-chave para o desenvolvimento permanente da qualidade da educação, uma vez que utilizam metodologia científica e informação objetiva para a tomada de decisão e a definição de políticas e práticas pedagógicas" [Lück 2009]. Nessa linha de pensamento, o Ciclo e-M 
apresentado no presente texto (vide seção 4) traz uma proposta cuja finalidade é favorecer o avanço da escola acerca da gestão da tecnologia no seu ambiente, como estratégia para alcançar melhor desempenho educacional.

O Ciclo e-M configura-se como suporte ao acompanhamento sistemático de autoavaliação da gestão tecnopedagógica no contexto escolar. Para fins desse estudo, o termo tecnopedagogia baseia-se na ideia de considerar que o ser tecnológico se forma através da tecnologia e que esta é inerente ao contexto do ser. Ou seja, as tecnologias, ou melhor, as tecnopedagogias ampliam o processo de formação do homem que é essencialmente tecnológico [Yanaze 2009].

Nesse viés, o acompanhamento da gestão tecnopedagógica torna-se uma condição necessária para o desenvolvimento de ações maduras e planejadas coletivamente quanto ao uso eficiente e pedagógico das Tecnologias da Informação e Comunicação - TIC, visto que estão cada vez mais presentes no cotidiano da escola.

Segundo dados recentes do Centro de Estudos sobre as Tecnologias da Informação e Comunicação [CETIC 2012], apesar de haver uma tendência positiva de aderência por iniciativa própria do professor ao uso de recursos móveis, a formação inicial docente ainda não integra de forma incisiva as novas tecnologias. Outro destaque na pesquisa do CETIC (2012) é identificação da crescente presença de computadores portáteis nas escolas públicas e a percepção de que a sala de aula se sobressai como local mais frequente de uso da tecnologia, no entanto, o uso das TIC em atividades pedagógicas ainda é instrumental e a velocidade de conexão limita o seu uso.

Nesse sentido, Passerino (2011) refere que os resultados de recentes pesquisas mostram que há um aumento significativo do acesso às TIC na nossa sociedade [...], que chegam ao espaço educativo, seja pela instalação de computadores nas escolas, seja pela instalação nos domicílios, e compreender essa nova realidade, bem como ela vem sendo gerenciada, é um desafio inerente aos educadores.

Esses dados levam a inferir que grande parte dos gestores e professores ainda não utiliza de forma satisfatória o potencial oferecido pelas tecnologias para melhorar seus resultados educacionais, ou as utiliza de forma bastante superficial, revelando uma necessidade de que sejam incorporadas ações pedagógicas ao cotidiano da escola, que colaborem para o alcance de melhores resultados educacionais. Para tanto, há necessidade de desenvolver uma postura autoavaliativa e planejada em relação ao uso educacional das TIC.

Esse novo perfil de educador faz emergir a necessidade de uma maturidade eletrônica (e-Maturity), que neste trabalho é conceituada como sendo "a evolução da capacidade e potencialidade da organização escolar de tomar decisões estratégicas e de utilizar de forma pedagógica e eficaz a tecnologia para melhorar os resultados educacionais" [Zednik et al 2012].

Nessa nova era digital, é fundamental que o gestor compreenda que a gestão eficiente da tecnologia pode potencializar o fazer pedagógico, e que a mesma também se caracteriza como condição necessária para redimensionar o fazer administrativo, de modo a facilitar a introdução de mudanças na gestão, na prática docente e na concepção pedagógica da escola. Nesse contexto, o papel do gestor exige uma nova visão: “[...] mais criativa, menos acomodada, mais participativa, mais ética, mais democrática e tecnologicamente mais exigente" [Alonso 2003] e proativa. 
Portanto, gerir a tecnologia na escola exige que o gestor compreenda as transformações tecnológicas, sociais e culturais em andamento, bem como as implicações dessas transformações para o desenvolvimento humano em sua plenitude. Isso requer considerar mudanças estruturais que vão muito além da simples introdução de computadores e internet numa escola. Exige a compreensão de que as TIC são meios de acesso e transformação da educação, do trabalho, do exercício da cidadania, do lazer... do mundo. Este é o entendimento que deve estar na base da gestão que deseja impulsionar o processo de $e$-Maturity, na visão redimensionada de gestão democrática, participativa, consciente e responsável.

O objeto de estudo deste artigo está centrado na apresentação e descrição das fases do Ciclo e-M. O estudo se justifica pela conveniência e importância de compreender o método: sua finalidade, composição e funcionamento, proposto na pesquisa de doutorado intitulada e-Maturity: gestão da tecnologia numa perspectiva de melhoria do desempenho pedagógico (PPGIE). O método utiliza uma abordagem teórico-analítica fundamentada no modelo $\mathrm{CM} 360^{\circ}$, proposto por Franco, Santos e Terra (2009), explicitada no tópico 3.

O artigo está organizado em cinco seções. Na seção 2, apresenta-se uma reflexão sobre a importância do ciclo de monitoramento da educação, relacionando-o à gestão tecnopedagógica. Na seção 3, descreve-se e analisa-se o aporte teórico que compõe o Ciclo e-M. A seção 4 apresenta e descreve o Ciclo e-M como proposta para avaliação sistemática da gestão tecnopedagógica. Finalmente, a seção 5 sintetiza as contribuições deste trabalho e apresenta sugestões para trabalhos futuros.

\section{Avaliação, Reflexão e Planejamento}

Este estudo incide na reflexão sobre o ciclo de monitoramento e sua relação com a $e$ Maturity, procurando evidenciar as implicações diretas que a construção dos ciclos tem na gestão tecnopedagógica e, consequentemente, na sua evolução. Isso pode ser observado nas mudanças que ocorrem em diferentes estágios de maturidade, demarcados por fases no decorrer da evolução da gestão de cada organização. "As mudanças se apresentam num contínuo, onde a evolução da imaturidade até a maturidade representa a personalidade sadia, e se desenvolve nesse contínuo" [Argyris 1968].

Num contexto educacional marcado pela constante modernização das TIC, a utilização do ciclo de monitoramento colabora para uma reflexão sistemática, cujas evidências encontradas a partir de uma prática reflexiva contribuem para 0 planejamento de ações coletivas que, por sua vez, contribuem para que a organização escolar possa tomar decisões estratégicas e passe a utilizar de forma mais efetiva e pedagógica a tecnologia para a melhoria dos resultados educacionais.

Nesta visão, o exercício de reflexão orientado por determinados instrumentos de trabalho que favorecem o desenvolvimento da capacidade de autoavaliação mostra quais as valências, as fragilidades e as dificuldades dos mesmos [Moreira e Ferreira 2011]. Nesse contexto, o Ciclo e-M se estabelece como instrumento de reflexão. Esse exercício pode colaborar com o processo de desenvolvimento da tríade Gestão, Tecnologia e Pedagogia.

O exercício da autoavaliação permite a um gestor e sua equipe escolar melhorarem o processo de ensino e aprendizagem com base na reflexão acerca do uso 
das TIC segundo 7 (sete) diferentes dimensões: Gestão e Liderança; Planejamento Curricular; Gestão do Processo de Ensino e Aprendizagem; Gestão da Avaliação; Formação Profissional para uso das TIC na Educação; Gestão dos Recursos e Gestão da e-Segurança.

Os elementos citados acima compõem a estrutura do questionário de autoavaliação do tipo web-based para aplicação no ambiente escolar, apresentado anteriormente no artigo e-Maturity (e-M): construção e elaboração do questionário de autoavaliação da Gestão Tecnopedagógica [Zednik et al 2013].

Os 6 (seis) primeiros elementos foram influenciados pelo Self Review Framework for ICT [Naace 2006] e adequados ao Modelo e-M. O sétimo elemento, esegurança ou e-safety, passou a compor o instrumental, tendo em vista que a função de avaliação e controle cumpre papel essencial num processo administrativo completo, já descrito por Fayol (1989), e que se caracteriza pela necessidade de orientar os estudantes e equipe escolar para compreenderem os riscos associados ao uso das TIC e à exposição à internet, bem como seu uso seguro, ético e consciente.

A proposta do Ciclo e-M visa apoiar e orientar o gestor no planejamento de ações necessárias de aprimoramento do uso pedagógico das TIC. Considera-se, ainda, que o Ciclo e-M pode ajudar a função de avaliação a assumir um papel essencialmente regulador, na medida em que serve de base para a reflexão da equipe escolar sobre todo o processo de uso das TIC em que está envolvida, proporcionando a mudança das suas práticas e concepções, através do acompanhamento de possíveis mudanças na dinâmica escolar da gestão tecnopedagógica, procedente do monitoramento do nível de $e$ Maturity. "Logo, o processo de avaliação se desdobra nas reflexões que levam à definição dos próximos passos de forma contundente e subsidiada pelos resultados e análise da avaliação" [Franco et al 2009].

Um ponto fulcral no Ciclo e-M é a autoavaliação, de caráter coletivo, e que traduz a apreciação que a equipe escolar, representada pelos diversos segmentos que compõem a escola, faz do seu próprio desempenho, resultado de uma postura reflexiva. Esta forma de "autorregulação considera a reflexão como estratégia autoavaliativa e a autoavaliação o verdadeiro vetor do desenvolvimento profissional, aquele que obriga à reflexão na e sobre a ação docente e reconstrói continuamente as suas práticas" [Moreira e Ferreira 2011]. Desta forma, se o desempenho escolar é o resultado do trabalho de toda uma equipe, o Ciclo e-M evidencia esse percurso evolutivo que deve ser visível com os avaliadores.

\section{Ciclo e-M: aporte teórico e aplicação}

O Ciclo e-M foi fundamentado e aperfeiçoado a partir do modelo CM360 proposto por Franco, Santos e Terra (2009), que é um framework de análise e avaliação de maturidade da gestão de conteúdo de uma organização.

O Ciclo e-M integra o modelo e-Maturity, que é um instrumental de análise do nível de maturidade, em relação ao uso estratégico e eficaz das TIC na melhoria dos resultados educacionais aplicado às escolas de Educação Básica. O instrumental encontra-se em fase de desenvolvimento pelo Grupo de Pesquisa em Modelagem Computacional Aplicada - GrPEC, da Universidade Federal do Ceará-UFC.

Nesse sentido, o instrumental colabora na identificação e acompanhamento dos usos e apropriações das TIC nas escolas, numa perspectiva de prática pedagógica e se 
consolida como instrumental de autoavaliação, mas também de acompanhamento e controle. O objetivo é, a partir de critérios avaliativos, produzir informações sobre o uso das TIC na escola, que auxiliem os gestores e comunidade escolar no planejamento, nas decisões e no estabelecimento de metas e implantação de ações pedagógicas e administrativas, visando à melhoria da qualidade do ensino e do rendimento escolar.

$\mathrm{Na}$ perspectiva de que "todos os modelos têm vantagens, limites e complementaridades. $\mathrm{O}$ mínimo de valores e de referência conceitual é necessário para se compreender o discurso universal da mudança" [Motta 2001], inicialmente será apresentado o modelo $\mathrm{CM} 360^{\circ}$, que embasou a concepção e estrutura do Ciclo e-M.

\subsection{Modelo CM360}

O modelo $\mathrm{CM} 360^{\circ}$ propõe uma escala de avaliação da maturidade para empresas, fundamentada em critérios pré-estabelecidos que permitem a organização e articulação de ações em consonância com os referidos critérios. O modelo foi "[...] desenvolvido primordialmente para servir como uma régua de avaliação de maturidade de uma organização sob critérios amplos e suficientes, que permitam a coordenação e alinhamento das suas ações relacionadas à gestão de conteúdo" [Franco et al 2009].

Embora o modelo $\mathrm{CM} 360^{\circ}$ seja voltado, a princípio, para a análise e avaliação de conteúdo, a ideia subjacente pode ser pensada em diferentes situações. Caracteriza-se como um modelo objetivo e abrangente, porém se diferencia por ser "uma ferramenta de análise do modelo organizacional de gestão do conteúdo, mas também um instrumento de planejamento e apoio para o desenvolvimento das competências e processos necessários a uma gestão eficaz" [Franco et al 2009].

Nessa proposta, a avaliação é o início do ciclo de monitoramento e evolução da gestão em uma organização. A aplicação do modelo é dividida em 4 (quatro) fases: preparação, avaliação, análise e ação, conforme diagrama abaixo.

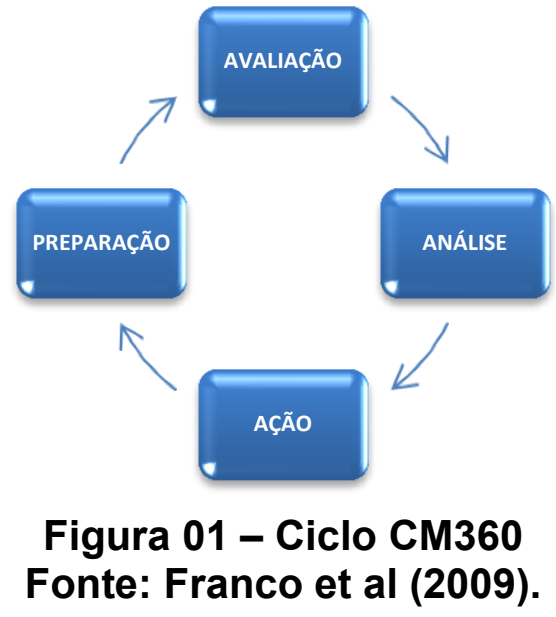

O ciclo inicia com a fase da preparação, onde há definição de facilitadores que farão o processo de avaliação. Em seguida, durante o processo de avaliação, os facilitadores devem selecionar as informações significativas para mencionar os níveis de maturidade de acordo com cada um dos 37 critérios (Quadro 01) estabelecidos em cada uma de suas quatro dimensões organizacionais. Após a avaliação, realiza-se uma análise qualitativa dos resultados, para que seja possível apontar possíveis mudanças e 
melhorias que serão consolidadas através de um plano de ação. O plano deve observar os objetivos e as estratégias da instituição.

Aplicando ao contexto educacional, o resultado do emprego deste modelo é que a avaliação pode ser estudada e analisada pelos diferentes segmentos da escola, permitindo a identificação de falhas e dificuldades e, a partir de uma análise, oferecendo condições para correção e aprimoramento contínuo, através da elaboração de planos de ação coletivos e colaborativos, isso fornece ao gestor uma visão de toda a escola. A continuidade do processo avaliativo permite que um novo processo se inicie ao final de cada ciclo de avaliação e aprimoramento, com o "intuito de avaliar a relevância das ações executadas e estabelecer um comparativo evolutivo entre os ambientes antes e depois do ciclo realizado" [Franco et al 2009].

Desta forma, o processo de avaliação ajuda a promover o alinhamento entre os participantes da instituição, no caso de uma escola, entre a equipe escolar e comunidade, aproximando-os através de uma linguagem comum e direcionando o olhar para os diversos aspectos da gestão, “[...] funcionando também como um processo de capacitação dos envolvidos. Unificam-se linguagens e conceitos e cria-se um ambiente favorável para mudança" [Franco et al 2009].

Assim, os autores atribuíram pilares, categorias e critérios para estruturar a ferramenta de avaliação, conforme mostra o Quadro 01.

\begin{tabular}{|c|c|c|}
\hline PILAR & CATEGORIA & CRITÉRIO \\
\hline \multirow{3}{*}{ ESTRATÉGIA } & Posicionamento estratégico & $\begin{array}{l}\text { Objetivos estratégicos; } \\
\text { Grupos de usuários prioritários; } \\
\text { Gestão de riscos; } \\
\text { Controle de informação; } \\
\text { Divulgação. }\end{array}$ \\
\hline & Governança e equipe & $\begin{array}{l}\text { Governança; } \\
\text { Competências; } \\
\text { Fluxos de trabalho. }\end{array}$ \\
\hline & Monitoramento de uso & $\begin{array}{l}\text { Testes de uso; } \\
\text { Mensuração do uso. }\end{array}$ \\
\hline \multirow{4}{*}{ INFORMAÇÃO } & Qualidade do conteúdo & $\begin{array}{l}\text { Público-alvo; } \\
\text { Tipos e formatos de conteúdo; } \\
\text { Acessibilidade; } \\
\text { Tradução e idiomas; } \\
\text { Articulaçãa do texto; } \\
\text { Formatação do texto. }\end{array}$ \\
\hline & Manutenção do conteúdo & $\begin{array}{l}\text { Gestão do ciclo de vida; } \\
\text { Fontes de dados; } \\
\text { Gestão de templates. }\end{array}$ \\
\hline & Arquitetura da informação & $\begin{array}{l}\text { Modelagem do conteúdo; } \\
\text { Taxonomia e metadados. }\end{array}$ \\
\hline & Serviços online & $\begin{array}{l}\text { Sistemas de atendimento; } \\
\text { Serviços de autoatendimento. }\end{array}$ \\
\hline \multirow{4}{*}{ INTERFACE } & Identidade corporativa & $\begin{array}{l}\text { Layout das páginas; } \\
\text { Look and feel. }\end{array}$ \\
\hline & Navegação & $\begin{array}{l}\text { Menus; } \\
\text { Modelos de página. }\end{array}$ \\
\hline & Página inicial & $\begin{array}{l}\text { Layout da página principal; } \\
\text { Marca e missão. }\end{array}$ \\
\hline & Busca & $\begin{array}{l}\text { Mecanismo de busca; } \\
\text { Interação com a busca }\end{array}$ \\
\hline
\end{tabular}




\begin{tabular}{|l|l|l|}
\hline \multirow{2}{*}{ TECNOLOGIA } & Desenvolvimento & $\begin{array}{l}\text { Flexibilidade e evolução; } \\
\text { Qualidade }\end{array}$ \\
\cline { 2 - 3 } & Operação & $\begin{array}{l}\text { Desempenho e disponibilidade; } \\
\text { Segurança. }\end{array}$ \\
\hline
\end{tabular}

\section{Quadro 01 - Organização hierárquica dos pilares, categorias e critérios do modelo $360^{\circ}$. Fonte: Franco et al (2009).}

Conforme apresentado no Quadro 01, pode-se perceber que o modelo integra design (a interface e tecnologia), conteúdo (informação e tecnologia) e negócios (estratégia e tecnologia), ou seja, a tecnologia é transversal a todas estas áreas, tornando-se essencial para criação de um produto de comunicação digital, bem como sua gestão.

O Quadro 02 apresenta os níveis e parâmetros de avaliação de maturidade do modelo $360^{\circ}$. Nesse modelo o processo de avaliação de maturidade apresenta um quadro de avaliação de desempenho gradativa que indica, quantitativamente, em qual etapa se encontra a iniciativa em questão. Os autores nomearam este indicador de nível de maturidade.

\begin{tabular}{|c|c|c|c|}
\hline \multicolumn{3}{|c|}{ DIMENSÃO } & PARÂMETRO DE AVALIAÇÃO \\
\hline \multirow{5}{*}{ 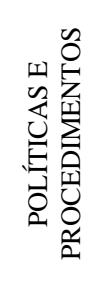 } & 1 & Não há & Não existe evidência. \\
\hline & 2 & Incipiente & Existem esboços informais. \\
\hline & 3 & Atende parcialmente & $\begin{array}{l}\text { Existe documentação plena das principais atividades relacionadas } \\
\text { à questão. }\end{array}$ \\
\hline & 4 & Atende plenamente & $\begin{array}{l}\text { Existe documentação plena } \quad \text { (abrangente } \mathrm{e} \text { revisada } \\
\text { continuamente) de todas as atividades relacionadas à questão. }\end{array}$ \\
\hline & 5 & Exemplar & São referência na questão. \\
\hline \multirow{5}{*}{ 秥 } & 1 & Não ciente & Não há percepção na equipe da relevância da questão. \\
\hline & 2 & Ciente & $\begin{array}{l}\text { Há percepção da relevância da questão, mas não há ação efetiva } \\
\text { ou há iniciativas não estruturadas. }\end{array}$ \\
\hline & 3 & $\begin{array}{l}\text { Parcialmente } \\
\text { acordado (a) }\end{array}$ & $\begin{array}{l}\text { Há processo de trabalho formal para as principais atividades } \\
\text { relacionadas à questão. }\end{array}$ \\
\hline & 4 & Institucionalizado (a) & $\begin{array}{l}\text { Há processo de trabalho formal para todas as atividades } \\
\text { relacionadas à questão. }\end{array}$ \\
\hline & 5 & Líder & Equipe e seus processos de trabalho são referência na questão. \\
\hline \multirow{5}{*}{ 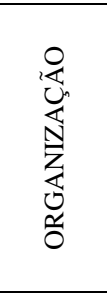 } & 1 & Não ciente & Não há percepção na organização de relevância da questão. \\
\hline & 2 & Ciente & $\begin{array}{l}\text { Há percepção de relevância da questão, mas não há ação efetiva } \\
\text { ou há iniciativas não estruturadas. }\end{array}$ \\
\hline & 3 & $\begin{array}{l}\text { Parcialmente } \\
\text { acordado (a) }\end{array}$ & $\begin{array}{l}\text { Apoia formalmente as principais atividades relacionadas à } \\
\text { questão. }\end{array}$ \\
\hline & 4 & Institucionalizado(a) & Apoia formalmente todas as atividades relacionadas à questão. \\
\hline & 5 & Líder & A questão é entendida como estratégia para a organização. \\
\hline \multirow{5}{*}{$\begin{array}{l}0 \\
0 \\
0 \\
0 \\
0 \\
0\end{array}$} & 1 & Não há & Não há solução para questão. \\
\hline & 2 & Incipiente & $\begin{array}{l}\text { Atende de forma desestruturada ou ad hoc a certas demandas } \\
\text { relacionadas à questão. }\end{array}$ \\
\hline & 3 & Atende parcialmente & $\begin{array}{l}\text { Atende plenamente às principais demandas relacionadas à } \\
\text { questão. }\end{array}$ \\
\hline & 4 & Atende plenamente & Atende plenamente a todas as demandas relacionadas à questão. \\
\hline & 5 & Exemplar & Solução praticada é referência na questão. \\
\hline
\end{tabular}

O Quadro 02 mostra que foi utilizada uma escala de 1 a 5 , que representa os níveis ou estágios, onde os parâmetros descrevem os critérios estabelecidos para 
avaliação em cada dimensão. "Esta régua é utilizada para todos os critérios de avaliação com o propósito de estabelecer parâmetros de maturidade transversais para o modelo" [Franco et al, 2009].

\section{Ciclo e-M}

A aplicação do questionário $\boldsymbol{e}$-Maturity: Gestão Tecnopedagógica de forma sistemática numa escola gera o Ciclo $e-M$, inspirado no modelo $\mathrm{CM} 360^{\circ}$; porém, neste estudo, faz-se uma diferença quanto à organização das fases, conforme apresenta a Figura 02, por considerar esta ordem mais adequada ao trabalho proposto.

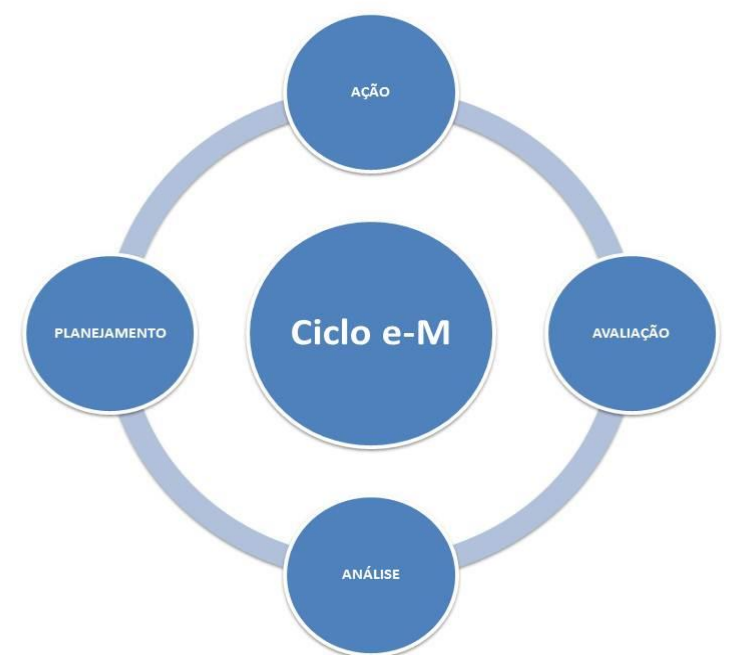

Figura 02 - Ciclo e-M

O Ciclo e-M se caracteriza por 4 (quatro) fases distintas, são elas: Ação, Avaliação, Análise e Planejamento.

A fase da Avaliação corresponde ao diagnóstico estruturado através do questionário de autoavaliação e-Maturity, onde os diversos segmentos que constituem a escola realizam uma avaliação coletiva baseada nas 7 (sete) dimensões apresentadas no tópico 2.

O Modelo $\mathrm{CM} 360^{\circ}$ destaca que "o processo de avaliação se desdobra nas reflexões que levam à definição dos próximos passos de forma contundente e subsidiada pelos resultados e análise da avaliação" [Franco et al, 2009].

A avaliação da maturidade tecnopedagógica da escola exige uma Análise reflexiva, detalhada, criteriosa e sistemática sobre o uso das TIC. "A reflexão sobre a ação e a reflexão sobre a reflexão na ação são fundamentais para o desenvolvimento do conhecimento profissional do professor" [Moreira e Ferreira, 2011] e, consequentemente, da equipe escolar. Segundo Korthagen e Wubbes (2001) a prática reflexiva contribui para que os participantes: “(a) valorizem a reflexão sobre a sua ação; (b) questionem as suas práticas; (c) identifiquem aspetos a melhorar; (d) analisem as suas relações".

Além das contribuições citadas por Korthagen e Wubbes (2001), é possível inferir que a análise oferece subsídios para o Planejamento de ações que objetivem 
introduzir as TIC de maneira holística na escola, ou seja, a partir da perspectiva sinérgica, onde os diferentes atores contribuem para uma ação coordenada e harmônica.

O planejamento deve prever intervenções coletivamente pensadas na realidade, para transformá-la. O planejamento, enquanto processo de previsão de necessidade, racionalização do uso dos recursos materiais e humanos disponíveis, organização e orientação das ações, tem a finalidade de "alcançar objetivos concretos, em prazos determinados e em etapas definidas a partir do conhecimento e avaliação científica da situação original" [Martinez e Lahone 1997].

A função do planejamento é tornar a Ação clara, precisa, eficiente, direcionada e transformadora. Desta forma, a Ação concretiza-se como resultado final de cada ciclo. Na medida em que contribui para uma reflexão na ação e, sobre esta, o Ciclo e-M impele a tomada de consciência das potencialidades e limitações e contribui para realizar a autoavaliação, ajuda na projeção de futuras mudanças e fortalece a ideia evolucionária de maturidade tecnológica na educação.

Segundo Argyris (1968) "quando se analisa a conduta humana em organizações, é difícil separar a diagnose da prognose". Portanto, é importante ressaltar que, para elaborar um plano de ação eficiente, é imprescindível a realização de um diagnóstico objetivo da realidade tecnopedagógica e, consequentemente, conjeturar as necessidades dos alunos e professores e as condições para atendê-las.

Franco et al (2009) ressaltam que uma análise identifica "anomalias aparentes e, ao ser realizado em detalhe, pode oferecer informações preciosas, indicando, por exemplo, uma ou mais dimensões que estejam mais aquém das demais, e isto pode ser o maior obstáculo para o amadurecimento de diversos critérios".

Com a finalidade de oferecer subsídios para a análise, o modelo e-Maturity classificou os níveis de maturidade, que serão emitidos em forma de feedback gerados pelo sistema do questionário web-based de autoavaliação, conforme apresenta o Quadro 03.

\begin{tabular}{|l|l|l|l|}
\hline \multicolumn{2}{|c|}{ NÍVEIS } & \multicolumn{1}{c|}{ PARÂMETRO DE AVALIAÇÃo } \\
\hline 1 & Leigo & Não existe evidência de ações planejadas ou desconhecem a questão. \\
\hline 2 & Receptivo & $\begin{array}{l}\text { Há percepção de relevância da questão, mas não há ação efetiva ou há } \\
\text { iniciativas isoladas, não estruturadas ou coordenadas. Os usuários estão } \\
\text { numa posição apenas receptiva da informação. }\end{array}$ \\
\cline { 2 - 4 } & 4 & Ciberconfluente & $\begin{array}{l}\text { Há processo de trabalho formal para as principais atividades relacionadas } \\
\text { à questão. }\end{array}$ \\
\hline 5 & Maduro & $\begin{array}{l}\text { É referência na apoia formalmente todas as atividades relacionadas à questão. } \\
\text { reorganizar as informações e transformá-las em conhecimento. }\end{array}$ \\
\hline
\end{tabular}

\section{Quadro 03 - Níveis e parâmetros de avaliação}

O mais importante, porém, não é simplesmente identificar o nível em que uma escola se encontra em relação uso pedagógico da tecnologia, mas a percepção de que o processo de autoavaliação possibilita um ciclo contínuo de reflexão e ação e, consequentemente, possibilita o desenvolvimento da $e$-Maturity. 


\section{Considerações Finais}

Este artigo buscou, através de uma abordagem teórico-analítica, apresentar e descrever as fases do Ciclo e-M. O modelo CM $360^{\circ}$ serviu como base teórica para fundamentar a proposta do Ciclo e-M, cujos contributos foram evidenciados ao longo do desenvolvimento do instrumental e aportados neste artigo.

O Ciclo e-M, descrito neste estudo, evidenciou o valor da reflexão sobre a avaliação com vista à construção situada do conhecimento acerca do uso criativo, estratégico e pedagógico das TIC no contexto escolar. Cada fase (Avaliação, Análise, Planejamento e Ação) vivenciada no ciclo permite a evolução sinérgica da maturidade tecnológica, apresentada no modelo e-Maturity - Gestão Tecnopedagógica.

Salienta-se que, em parceria com a Universidade Federal do Ceará - UFC, através do Grupo de Pesquisa em Modelagem Computacional Aplicada - GrPEC, o sistema do questionário web-based encontra-se em fase de desenvolvimento. Em trabalhos futuros pretende-se descrever o desenvolvimento e funcionamento do sistema.

\section{Referências}

Alonso, Myrtes (2003). “A gestão/administração educacional no contexto da atualidade". In: Gestão Educacional e Tecnologia. São Paulo: Avercamp.

Argyris, C. (1968). "Personalidade e organização: o conflito entre o sistema e o indivíduo". Rio de Janeiro: Renes.

CETIC (2013). "Pesquisa TIC Educação 2012: Pesquisa sobre o uso das TIC nas escolas brasileiras”. São Paulo: Centro Regional de Estudos para o Desenvolvimento da Sociedade da Informação. Disponível em $<$ http://www.cetic.br/educacao/2012/apresentacao-tic-educacao-2012.pdf $>$ Acesso em: 10/08/2013.

Fayol, Henri. Administração industrial e geral: previsão, organização, comando, coordenação, controle. São Paulo: Atlas, 1989.

Franco, Carlos Eduardo; Santos, Marcelino Luis B.; Terra, José Cáudio C. (2009) "Gestão de conteúdo $360^{\circ}$ : integrando negócios, design e tecnologia". São Paulo: Saraiva.

Korthagen, F. e Wubbels, T. (1995). "Characteristics of Reflective Practitioners: Towards an Operationalization of the Concept of Reflection". Teachers and Teaching: theory and practice, $1,1,51-72$

Lück, Heloísa (2009). "Dimensões da gestão escolar e suas competências". Curitiba: Editora Positivo.

Moreira, Jacinta Rosa e Ferreira, Maria José (2011). "Webfolios reflexivos: contributos para o desenvolvimento profissional do professor". Revista EFT: <http://eft.educom.pt.>Educação, Formação \& Tecnologias.

Martinez, M.J. Lahone, C.O. (1997). “Planejamento escolar”. São Paulo: Saraiva.

Motta, Paulo Roberto. (2001) “Transformação organizacional: a teoria e a prática de inovar". Rio de janeiro: Qualitymark. 
NAACE (2006). "Self-review Framework (SRF)". Universidade de Nottingham. Acesso em: 03 de março de 2012. Disponível em: <http://www.naace.co.uk/ictmark/srf>.

Passerino, Liliana Maria (2011). "Políticas públicas e novas tecnologias: a nova roupa do rei?" In: Ações institucionais de avaliação e disseminação de tecnologias educacionais. 1 ${ }^{\mathrm{a}}$. Edição. Porto Alegre: JSM Comunicação.

Yanaze, Leandro Key Higuchi (2009) "Tecno-pedagogia: os aspectos lúdicos e pedagógicos da comunicação digital". Dissertação de mestrado. São Paulo: Universidade de São Paulo - Escola de Comunicação e Artes.

Zednik, Herik; Tarouco, Liane M. R. e Klering, Luis R. (2012) "E-Maturity: entrelaçando gestão, tecnologia e pedagogia". Porto Alegre: Revista Renote - Novas Tecnologias na Educação, V. 10 N 3, dezembro/2012.

Zednik, Herik; Tarouco, Liane M. R. e Klering, Luis R. (2013) "E-maturity (e-M): construção e elaboração do questionário de autoavaliação da Gestão Tecnopedagógica". Porto Alegre: Revista Renote - Novas Tecnologias na Educação, V. $11 \mathrm{~N}^{\mathrm{o}} 1$, julho/2013. 